\title{
Envolvimento cognitivo de universitários em relação à motivação contextualizada
}

\author{
Daniella Maculan Pavesi Accorsi - Universidade Estadual de Londrina \\ José Aloyseo Bruneck - Universidade Estadual de Londrina' \\ Sueli Édi Rufini Guimarães - Universidade Estadual de Londrina
}

\begin{abstract}
Resumo
O presente estudo propôs-se investigar aspectos motivacionais de 217 alunos de um curso superior de sete diferentes instituições. Os participantes foram avaliados quanto à crença no valor da inteligência e do esforço, quanto às suas metas de realização e quanto ao uso de estratégias de aprendizagem usadas numa disciplina teórica específica. Como resultados, apareceu maior incidência da orientação à meta aprender e correlação negativa entre essa orientação e a orientação à meta evitação do trabalho. Ao mesmo tempo, a orientação à meta aprender esteve mais associada ao uso de estratégias de profundidade, enquanto a preferência por estratégias de superfície esteve mais associada à meta evitação do trabalho. O esforço não apareceu como fator mais valorizado do que a inteligência por grande parte da amostra. Os resultados foram discutidos à luz da literatura atual e foram extraídas conclusões para o ensino superior.

Palavras-chave: Motivação no ensino superior; Estratégias de aprendizagem; Valorização do esforço; Valorização da capacidade.
\end{abstract}

\section{University students' cognitive involvement in relation to situated motivation}

\begin{abstract}
The aim of the present study was to investigate 217 university students' motivational orientations from seven different schools. Participants answered questionnaires that asked their beliefs about effort value versus ability in school achievement contexts, their achievement goal orientations, and learning strategies they use in the study of a specific subject matter. Results showed that students adopted more learning than work avoidance goal, and theses two orientations were negatively related. At the same time learning goal was significantly more related to deep strategies' use while work avoidance goal was related to surface strategies. Nevertheless effort was not considered by most students as more important than ability. Results were discussed in the light of the current literature and some conclusions were drawn for teaching in higher education.
\end{abstract}

Keywords: Motivation in higher education; Learning strategies; Value of effort versus ability.

\section{Introdução}

Encontra-se, no ensino superior, considerável variação na qualidade do envolvimento nos estudos entre alunos de cursos e instituições diversas, entre alunos de um mesmo curso e, até numa mesma turma e disciplina. Entende-se aqui por qualidade do envolvimento, mais do que a intensidade do esforço ou dedicação, o seu direcionamento, ou seja, no que precisamente o aluno investe suas capacidades e o tempo, durante as atividades acadêmicas. Em outras palavras, e mais especificamente, esse envolvimento é de natureza cognitiva e consiste no uso de estratégias de aprendizagem (Greene \& Miller, 1996; Weinstein \& Mayer, 1986).

Brevemente, estratégias de aprendizagem são ações mentais e comportamentos que os alunos utilizam

${ }^{1}$ Endereço para correspondência:

Rua Rui Barbosa, 187 - J. Tatiani - 86070-610 - Londrina-PR

E-mail: bzuneck@sercomtel.com.br durante a aprendizagem e que influenciam esse processo e seus resultados (Weinstein \& Mayer, 1986). Tais estratégias dividem-se em categorias e táticas mais específicas, cujas taxionomias variam conforme os autores (por exemplo, Pintrich \& Garcia, 1991; Weinstein \& Mayer, 1986). Entretanto, tem sido amplamente considerada na literatura a distinção mais inclusiva entre estratégias de profundidade e de superfície, originada de uma versão teórica que explica os processos de aprendizagem como processamento da informação (para uma explanação mais ampla, ver Bzuneck, 2004a). Autores como Craik e Lockhart (1972), entre outros, propuseram que melhores resultados nas aprendizagens são obtidos quando o nível de processamento for de profundidade, ou seja, quando o trabalho mental do aluno consistir de busca de significado, com preocupação pela compreensão, mediante o recurso 
aos conhecimentos prévios. Em contraste, o processamento de superfície restringe-se a algo equivalente ou muito próximo à simples reprodução dos conteúdos, sem verdadeira compreensão. A esses dois níveis de processamento da informação correspondem as duas categorias de estratégias de aprendizagem - de profundidade ou de superfície (Biggs, Kember \& Leung, 2001; Pintrich \& Garcia, 1991). Um envolvimento nos estudos com estratégias de profundidade conduzirá a resultados finais de aprendizagem muito superiores em comparação com o emprego de estratégias de superfície (Greene, Miller, Crowson, Duke \& Akey, 2004).

Pintrich e Garcia (1991), ao considerarem explicitamente o contexto do ensino superior, enfatizaram que componentes motivacionais são inerentes à preferência e uso de certas estratégias nos processos de aprendizagem, isto é, a adoção por parte dos alunos de estratégias de profundidade ou de superfície dependerá dos seus objetivos ou metas como fator motivacional. Esses autores consideraram duas orientações motivacionais opostas, que denominaram intrínseca e extrínseca, com suas respectivas influências sobre a escolha de estratégias. Alunos que têm por objetivo uma compreensão dos conteúdos (orientação intrínseca) adotam estratégias de profundidade, enquanto os interessados apenas em terminar as atividades, conseguir boas notas, aparecer como melhores os colegas etc. (orientação extrínseca) contentam-se com estratégias superficiais.

Ainda segundo Pintrich e Garcia (1991), as orientações motivacionais intrínseca e extrínseca têm estreita correspondência com aquilo que outros teóricos denominavam metas aprender e performance (Elliott \& Dweck, 1988) ou metas tarefa e ego (Nicholls, 1984). Essa terminologia ficou consagrada nos estudos que têm adotado uma abordagem contemporânea denominada Teoria de Metas de Realização. Com esse enfoque teórico realizaram-se inúmeros estudos que tratam das estratégias de aprendizagem. Por isso, ele será aqui brevemente exposto.

Metas de realização referem-se a um conjunto de pensamentos, propósitos, percepções, crenças, atribuições e conceitos que, por sua vez, levam a conseqüências tanto cognitivas quanto afetivas e comportamentais. Em síntese, essas metas são orientações abrangentes que vão explicar por que o aluno se envolve em determinada tarefa e busca atingir determinados objetivos acadêmicos (Ames, 1992; Bzuneck, 2004b; Urdan, 1997).

Inicialmente, foram consideradas nos estudos duas metas de realização, a saber, a meta denominada alternativamente de aprender, ou domínio, ou tarefa, e a meta performance ou ego (Elliott \& Dweck, 1988; Nicholls, 1984). Os alunos que adotam a meta aprender (domínio, tarefa) caracterizam-se por visar dominar os assuntos, ampliar conhecimentos, melhorar habilidades, enfim, crescer intelectualmente. Para tanto, valorizam sobremaneira o esforço pessoal e costumam utilizar as melhores estratégias de aprendizagem diante dos desafios. Já alunos com orientação à meta performance ou ego revelam especial preocupação com a própria inteligência. Dessa forma, conforme pesquisas mais recentes, existem, alunos mais preocupados com mostrar-se inteligentes, ser os primeiros (meta performance-aproximação), enquanto outros visam apenas não aparecer como incompetentes ou incapazes (meta performance-evitação). A cada um dos dois casos estão associados comportamentos diferenciados em relação a empenho, uso de estratégias e de níveis de processamento.

Por fim, uma quarta meta foi proposta por Archer (1994), denominada alienação acadêmica ou evitação do trabalho, e contemplada inicialmente em estudos com universitários. Ela qualifica um determinado acadêmico cujo objetivo é apenas executar as tarefas acadêmicas com o mínimo de esforço, tanto que realização para ele é atingir êxito sem grande aplicação nos estudos. Note-se que essa meta difere da orientação à performanceevitação, em que o esforço não é desvalorizado e a evitação refere-se apenas à condição de aparecer como incapaz. De acordo com resultados de pesquisas, aluno com meta alienação acadêmica usa estratégias pobres nos estudos, prefere tarefas que podem ser completadas facilmente e em pouco tempo ou que simplesmente não exijam trabalho duro (Archer, 1994; Meece, 1991; Seifert \& O'Keefe, 2001; Wolters, 2003). Como esperado, Harackiewicz, Barron, Elliot, Carter e Lehto (1997) e Wolters (2003), entre outros, comprovaram que uma acentuada orientação para essa meta se relaciona com baixos resultados acadêmicos.

Os proponentes e seguidores da teoria de metas de realização (ver Ames, 1992; Bzuneck, 2004b; 2005) explicam igualmente como se formam tais metas nas pessoas, ou seja, de que fatores elas dependem. A adoção de cada uma dessas metas ocorre de modo acentuado, embora não exclusivamente, em razão das percepções que os alunos têm do seu contexto de aprendizagem. O contexto percebido concentra-se na figura do(a) professor(a) com suas ações, reações, verbalizações, formas de avaliação, em suma, com seu modo de conduzir a disciplina e de interagir em classe. Nas pesquisas que adotaram esse referencial teórico, os autores comprovaram a relação entre tais percepções com a adoção de uma meta de realização e, como conseqüência, com a preferência por determinadas estratégias. A título de amostra, Church, Elliot e Gable (2001) consideraram como as aulas eram vistas por alunos de um curso superior de química, quanto ao grau com que elas cativavam interesse, a intensidade da

Psico-USF, v. 12, n. 2, p. 291-300, jul./ dez. 2007 
valorização das notas e o rigor das avaliações. Os resultados comprovaram os pressupostos teóricos. Relações idênticas apareceram em estudos brasileiros, também com alunos de cursos superiores (entre outros, Cardoso \& Bzuneck, 2004; Stefano, Passador \& Bzuneck, 2003; Zenorine \& Santos, 2003).

Em síntese, os dados de pesquisa têm conferido apoio à conclusão de que alunos do ensino superior podem ter envolvimento de maior ou menor qualidade nos estudos, quanto às estratégias de aprendizagem que costumam empregar. A preferência por estratégias, por sua vez, está associada a uma determinada orientação motivacional ou meta de realização. E o ambiente psicológico de sala de aula, enquanto percebido pelos alunos, é o determinante principal da adoção de cada meta, ou seja, a motivação é sempre contextualizada.

Da breve descrição apresentada anteriormente de cada meta de realização, conclui-se que um contraste marcante entre alunos voltados à meta aprender e alunos identificados pela evitação do trabalho ocorre em virtude do apreço diferenciado pelo esforço. Entretanto, estudos brasileiros como o de Bzuneck e colaboradores (2003) e o de Cardoso e Bzuneck (2004) descobriram, nas respectivas amostras, altos escores médios tanto na meta evitação do trabalho como na meta aprender, o que parece estar em desacordo com outras pesquisas que concluíram por correlações negativas entre os escores nessas duas metas (Archer, 1994). Adicionalmente, como o sugerem Vermetten, Lodewijks e Vermunt (2001), devem-se focalizar explicitamente, nos instrumentos de medida da meta aprender, tanto o objetivo de aumentar as competências como a valorização do esforço. A ausência de avaliação explícita dessa última variável pode levar a resultados que não correspondam ao conceito teórico desse construto. Por sua vez, a avaliação da meta evitação do trabalho deverá conter maior número e variedade de itens para se identificar mais adequadamente essa orientação motivacional nos alunos. Por essas razões, o presente estudo limitou-se a avaliar apenas essas duas metas de realização, focalizando explicitamente a valorização do esforço nas orientações motivacionais de alunos de cursos superiores. Mais especificamente, buscou-se atingir os objetivos seguintes:

1. Avaliar padrões motivacionais de alunos de um curso superior em relação a uma disciplina teórica e importante. Como padrões motivacionais, foram selecionadas apenas duas metas de realização aprender e evitação do trabalho -, conceitualmente opostas quanto à valorização do esforço.

2. Verificar a crença dos alunos sobre o peso da inteligência ou aptidão e do esforço nos resultados acadêmicos, e comparar tal crença com as metas de realização.

3. Identificar aspectos contextuais da motivação, ou seja, como os alunos percebem o grau de exigências nas avaliações e a carga de trabalho na disciplina, e relacionar essas percepções com as metas de realização.

4. Conhecer as estratégias de aprendizagem - de superfície ou de profundidade - que os alunos alegam utilizar na disciplina e relacioná-las com a adoção de metas de realização.

\section{Metodologia}

\section{Amostra}

Participaram deste estudo 217 alunos do curso de nutrição de sete instituições de ensino superior do Paraná. Desse total, 216 eram mulheres e somente um homem, fato comum nessa carreira. As idades variaram de 19 a 50 anos, com uma média de 23 anos. Uma condição para ser incluídos na pesquisa é que estivessem matriculados na disciplina de nutrição e saúde pública, alocada na terceira série em todas as referidas instituições. Essa disciplina específica foi selecionada como referencial para os autorelatos dos alunos, por ser de natureza teórica e cobrar leitura constante de textos científicos, e de jornais e revistas, além de exigir que o aluno faça resumos por escrito e trabalhe com acontecimentos do dia-a-dia, relacionando-os aos conteúdos teóricos. De resto, a consideração de apenas uma disciplina como foco atende à observação de vários autores, como Jacobs e Newstead (2000) e Pintrich (2000), de que varia, conforme a disciplina, tanto a orientação motivacional de cada aluno como a qualidade de seu envolvimento por meio do uso de estratégias de estudo.

\section{Instrumentos de medida}

Em atenção aos objetivos do estudo, por primeiro, após a solicitação de preenchimento dos dados demográficos, procurou-se saber, através de uma única questão, como os alunos valorizavam, comparativamente, a inteligência ou aptidão e o esforço para os resultados positivos nos estudos. Para isso, eles deveriam assinalar que peso atribuíam, em valores de porcentagem, a cada uma dessas duas variáveis, esclarecendo-se que o somatório de ambas seria 100.

Seguiu-se um questionário de três partes em escala Likert de cinco pontos, desde 1 (nada verdadeiro) até 5 (totalmente verdadeiro), destinadas a avaliar as duas metas de realização, as percepções do contexto e o uso de estratégias de aprendizagem. Alguns itens incluídos nessas escalas foram extraídos de outros instrumentos, constantes da literatura, já traduzidos e adaptados para pesquisas 
brasileiras (Bzuneck e colaboradores, 2003; Cardoso \& Bzuneck, 2004). Outros itens foram criados com base nos construtos teóricos a serem avaliados e alguns, disponíveis na língua inglesa, foram traduzidos. A tradução para o português foi feita por um dos autores da pesquisa e, em seguida, outro pesquisador com bastante domínio da língua inglesa realizou a versão do questionário para o inglês (back translation), sendo então comparada com o original em inglês. As discrepâncias percebidas foram verificadas e discutidas, resultando na tradução definitiva. Segue-se uma breve descrição dos procedimentos de construção dessas escalas.

\section{Escala de Metas de Realização}

Foram elaborados 23 itens destinados a avaliar a adoção das metas aprender e evitação do trabalho. Entre os 11 itens relativos à meta aprender foi dada ênfase à valorização do esforço, nem sempre explicitada em instrumentos anteriores. Assim, para a presente pesquisa construíram-se cinco itens novos relativos a esta meta, como, por exemplo: "a melhor maneira de me dar bem nesta disciplina é me esforçar muito". Os restantes seis itens já tinham sido utilizados em pesquisas brasileiras anteriores (Bzuneck e colaboradores, 2003; Cardoso \& Bzuneck, 2004). O índice de consistência interna desse conjunto de itens foi de 0,69 pelo alfa de Cronbach.

Já para investigar a meta evitação do trabalho, utilizaram-se 12 itens, tendo como referência instrumentos anteriores como o de Biggs e colaboradores (2001), e Seifert e O'Keefe (2001). Um exemplo deste item é: “eu prefiro disciplinas em que não se tenha que trabalhar duro" (alfa $=0,75)$.

\section{Escala de Avaliação das Percepcôes de Variáveis de Contexto}

Como foi sugerido por literatura específica (Church e colaboradores, 2001; Marsh, 2001), duas variáveis de contexto percebido foram consideradas nesta escala: o grau de exigências das avaliações, com quatro itens (alfa $=0,49)$, e a carga de trabalho na disciplina, com três itens (alfa $=0,64)$. As afirmativas "os critérios de notas nesta disciplina são muito difíceis de satisfazer" e "o professor desta disciplina passa tanto trabalho que não tenho tempo para mais nada" são exemplos dessas duas subescalas, respectivamente.

\section{Escala de Avaliação de Estratégias de Aprendiragem}

Os alunos deveriam assinalar em que grau utilizam, nos estudos daquela disciplina, estratégias de superfície, com oito itens, ou estratégias de profundidade, com 13 itens (Bzuneck e colaboradores, 2003; Cardoso \& Bzuneck, 2004; Church e colaboradores, 2001). São exemplos dessas estratégias questões como "eu aprendo os conteúdos decorando, mesmo que não os entenda" (estratégia de superfície) e "eu estudo cada tópico da disciplina de tal modo que eu possa formar minhas próprias conclusões" (estratégia de profundidade). Os valores de consistência interna foram, respectivamente, 0,80 e 0,67 pelo alfa de Cronbach.

Antes da aplicação à amostra final, o instrumento foi testado com 24 alunas de um curso de nutrição que já haviam passado pela disciplina de nutrição e saúde pública. Essa aplicação ocorreu em duas etapas. Num primeiro momento, o questionário foi aplicado a apenas quatro alunas desse grupo. $\mathrm{Na}$ ocasião, a primeira autora leu cada questão e as alunas, além de marcar na escala, eram solicitadas a apontar eventuais dúvidas em relação ao enunciando e à pertinência das questões em relação à disciplina em foco. Foram levantados poucos problemas nos enunciados e descobertas algumas questões repetidas. $\mathrm{O}$ mesmo questionário piloto, já aperfeiçoado em função dessa primeira experiência, foi aplicado novamente a outros 20 alunos. Como resultado, não surgiu qualquer problema com o enunciado das frases. Com base nas duas aplicações do questionário piloto, concluiu-se pela necessidade de se retirar duas questões repetidas e corrigir inadequações de alguns termos, chegando-se assim à redação definitiva, que foi utilizada com a amostra principal.

\section{Procedimentos de coleta de dados}

Para a coleta de dados junto à amostra definitiva, foram inicialmente contatados por telefone os coordenadores de sete entre os 21 cursos de nutrição espalhados por quatro grandes cidades do Paraná, incluindo a capital, nas quais se encontram sete desses cursos. Todos os 21 são reconhecidos pelo MEC e somente um pertence a universidade pública. Embora selecionados por conveniência, atendeu-se ao critério de que os sete cursos da amostra deveriam representar todas as regiões do Estado.

O primeiro contato com os coordenadores ensejou que fosse agendada uma visita a cada instituição pela segunda autora do trabalho. Em hora de aula, e com o consentimento da respectiva professora, ela foi apresentada aos alunos e, a seguir, passou a informá-los dos objetivos gerais da pesquisa, solicitando sua colaboração em responder ao questionário, que seria de forma anônima, sem qualquer possibilidade de identificação das pessoas. Foi explicitado que se alguém não o quisesse fazer, não haveria nenhum problema. Somente na universidade pública duas alunas negaram-se a preencher o questionário e retiraram-se da sala. No restante das instituições não houve nenhuma recusa. 


\section{Resultados}

\section{Estatística descritiva}

Por primeiro, serão apresentados os aspectos motivacionais dos alunos, expressos na orientação às duas metas de realização investigadas, complementados pela avaliação de sua crença sobre a importância do esforço versus inteligência para o rendimento acadêmico.

A média de todos os alunos dos sete cursos $(\mathrm{N}=217)$ na meta aprender foi de 3,43 (DP=0,78), com variação entre os cursos de 3,17 (DP=0,73) a 3,80 (DP=0,67). Na meta evitação do trabalho, a média foi de 1,87 (DP=0,68), variando entre $1,70(\mathrm{DP}=0,59)$ e 2,18 $(\mathrm{DP}=0,69)$. Configura-se, portanto, a tendência da amostra total adotar mais a meta aprender.

Um outro objetivo deste trabalho foi verificar a crença dos alunos em relação ao peso da inteligência, capacidade (ou genericamente o QI), em comparação com o esforço para a performance acadêmica. A atribuição de mais de $50 \%$ a uma das variáveis indicaria que para aquele aluno essa era a variável mais crucial. A marcação de $50 \%$ para cada uma revelaria que ambas teriam peso igual. Dos 209 alunos que responderam à questão, 50 (23,9\%) apontaram que a inteligência tem mais peso nas aprendizagens escolares e $61(29,2 \%)$, que é o esforço a variável mais importante. Já para os restantes 98 (46,9\%), ou seja, para quase a metade da amostra, ambas contribuem com o mesmo peso.

Quanto a variáveis de contexto, a média na análise da percepção de que as avaliações na disciplina são exigentes foi de 2,59 (DP=0,78), oscilando entre os cursos desde uma média de 2,20 ( $\mathrm{DP}=0,65)$ até 3,31 ( $\mathrm{DP}=0,77)$. Semelhantemente, na medida da percepção do peso da carga de trabalho naquela disciplina, a média foi 2,06 $(\mathrm{DP}=0,90)$ e as médias intergrupais ficaram entre 1,60 $(\mathrm{DP}=0,63)$ e 3,08 $(\mathrm{DP}=1,00)$.

Por último, em relação ao uso de estratégias de aprendizagem de profundidade, a média geral foi 2,93 (DP=0,64), com variação de 2,69 (DP=0,65) até 3,31(DP=0,57). Já na avaliação do uso de estratégias de superfície, a média geral encontrada foi 2,53 (DP=0,65), com variações entre 2,33 (DP=0,62) e 2,85 (DP=0,70).

\section{Relações entre as variáveis}

Primeiramente, com o escopo de verificar a relação entre as crenças na capacidade e no esforço com a adoção das metas de realização, foi aplicado o teste do $\chi^{2}$ sobre a amostra total. Como critério da valorização da capacidade ou do esforço estabeleceu-se que o aluno tenha marcado acima de 50\% em um deles. Isto é, foram contemplados apenas os casos de valorização acentuada de cada uma das variáveis. Além disso, foram considerados aqueles alunos com escores mais altos $(\geq 2,50)$ quer na meta aprender quer na meta evitação do trabalho. Dessa forma surgiram quatro conjuntos de alunos: (a) altos escores na meta aprender e alta valorização da capacidade; (b) altos escores na meta evitação do trabalho e alta valorização da capacidade; (c) altos escores na meta aprender e valorização do esforço; e (d) altos escores na meta evitação do trabalho e alta valorização do esforço. Essa distribuição pode ser visualizada na Tabela 1.

Tabela 1 - Comparação entre a crença no QI e esforço e adoção de metas de realização

\begin{tabular}{lcc}
\hline & Alta meta aprender & Alta meta evitação do trabalho \\
& $(\geq 2,50)$ & $28(18,90)$ \\
\hline Valorização da capacidade & $41(27,7 \%)$ & grupo B \\
Valorização do esforço & grupo A & $23(15,5 \%)$ \\
& $56(37,8 \%)$ & grupo D \\
\hline
\end{tabular}

Duas associações significativas foram encontradas. Entre o grupo A (escores altos na meta aprender e na valorização da capacidade) e o grupo D (escores altos na meta evitação e valorização do esforço) surgiu um $\chi^{2}=4,52(p=0,03)$, o que indica tratar-se de grupos distintos e definidos. Por outro lado, entre os grupos B (altos escores na meta evitação e valorização do QI) e C (altos escores na meta aprender e valorização do esforço) encontrou-se um $\chi^{2}=8,68(p=0,00)$. Buscou-se também descobrir a relação entre as orientações às metas de realização adotadas pelos alunos e a percepção de variáveis do contexto (avaliação exigente e carga de trabalho). Os dados constantes da Tabela 2 demonstram uma correlação positiva e significativa entre a meta evitação do trabalho e as duas variáveis percebidas do ambiente (carga de trabalho e avaliação exigente). Entretanto, a meta aprender demonstrou uma correlação negativa e significativa com a percepção de carga de trabalho e correlação zero com a percepção de avaliação exigente. 
Tabela 2 - Correlações de Pearson entre adoção de metas de realização e as variáveis do ambiente de aprendizagem percebido

\begin{tabular}{lcccc}
\hline & \multicolumn{2}{c}{ Meta aprender } & \multicolumn{2}{c}{ Meta evitação do trabalho } \\
& $\mathrm{r}$ & $\mathrm{p}$ & $\mathrm{r}$ & $\mathrm{p}$ \\
\hline Avaliação exigente & 0,0280 & 0,686 & 0,1998 & 0,004 \\
Carga de trabalho excessiva & $-0,3036$ & 0,000 & 0,5166 & 0,000 \\
\hline
\end{tabular}

O teste de correlação de Pearson foi também utilizado para se identificar a relação entre a adoção de metas e o uso de estratégias de aprendizagem. Os resultados expostos na mesma Tabela 3 demonstram uma correlação positiva e significativa entre o uso de estratégias de profundidade e a adoção da meta aprender, bem como entre as estratégias de superfície e a meta de evitação do trabalho. Já as correlações entre o uso de estratégias de superfície e adoção da meta aprender e entre as estratégias de profundidade e a meta de evitação do trabalho foram negativas e significativas.

Tabela 3 - Correlações de Pearson entre adoção de metas e estratégias de aprendizagem

\begin{tabular}{lcccc}
\hline & \multicolumn{2}{c}{ Meta aprender } & \multicolumn{3}{c}{ Meta evitação do trabalho } \\
& $\mathrm{r}$ & $\mathrm{p}$ & $\mathrm{r}$ & $\mathrm{p}$ \\
\hline Estratégias de profundidade & 0,5491 & 0,000 & $-0,3739$ & 0,000 \\
Estratégias de superfície & $-0,3317$ & 0,000 & 0,4775 & 0,000 \\
\hline
\end{tabular}

Por meio da análise de regressão, buscou-se identificar se a adoção de metas de realização é preditiva do uso de estratégias de aprendizagem. A adoção da meta aprender prediz positivamente estratégias de profundidade como variável dependente $(\beta=0,55 \mathrm{p}=0,00)$ e tem razão negativa de predição sobre estratégias de superfície $(\beta=$ $-0,33 \mathrm{p}=0,00)$. Por outro lado, a adoção da meta de evitação de trabalho prediz negativamente as estratégias de profundidade $(\beta=-0,36 \mathrm{p}=0,00)$ e, positivamente, as de superfície $(\beta=0,47 \mathrm{p}=0,00)$.

\section{Discussão}

O presente estudo traz descobertas que podem contribuir para a compreensão da motivação de alunos em cursos superiores, bem como para a elaboração de medidas de avaliação desse construto. Descritivamente, os resultados mostraram uma tendência da amostra como um todo de adotar a meta aprender em relação a uma disciplina específica, o que faz prever maior envolvimento e, conseqüentemente, o desejável aumento de conhecimentos e habilidades. Ao mesmo tempo, foram mais baixos os escores na meta evitação do trabalho do que na meta aprender. Nesse último caso, houve diferença em relação aos resultados em outros estudos brasileiros como no de Bzuneck e colaboradores (2003), Cardoso e Bzuneck (2004), Stefano e colaboradores (2003), que haviam encontrado maior proximidade entre os escores médios na meta evitação do trabalho e na meta aprender. Por hipótese, é possível atribuir esses últimos resultados a problemas nos instrumentos de mensuração que o presente estudo tentou superar.
A literatura (por exemplo, Archer, 1994; Seifert \& O'Keefe, 2001) atesta que a variável crítica na discriminação entre meta aprender e evitação do trabalho é a valorização do esforço. Entre os procedimentos deste estudo esteve a criação de itens que explicitassem o esforço como condição para adoção da meta aprender, visando discriminar os alunos que dizem querer adquirir novos conhecimentos sem, na verdade, aplicar muito esforço, daqueles que se dispõem a contribuir de maneira ativa para a aquisição dos conhecimentos. As escalas da meta aprender e da meta evitação do trabalho atenderam a esse critério e, por suas propriedades psicométricas, constituem um instrumento válido de medida dessas duas metas de realização como possível referencial em pesquisas futuras.

De acordo com a teoria de metas de realização (Ames, 1992; Bzuneck, 2004b), alunos valorizam o esforço ou a inteligência de modo diferente, o que os faz se encaixarem no perfil de diferentes metas de realização. Assim, no presente estudo, buscou-se identificar, através de uma questão específica, em que grau alunos voltados a uma ou outra das duas metas de realização consideram de mais valor para os resultados acadêmicos o esforço ou a inteligência, mediante uma questão específica. Os resultados deveriam corroborar a estatística descritiva a respeito das orientações às duas metas. Entretanto, com a presente amostra, surgiram algumas especificações.

A maioria dos acadêmicos, independentemente da orientação motivacional, respondeu que acredita que o esforço e a inteligência têm o mesmo peso para os resultados escolares. Além disso, quando se formaram grupos extremos, isto é, de alunos com altos escores na 
meta aprender ou na meta evitação do trabalho, descobriuse que, dentro do grupo com mais altos escores na meta aprender, existem tanto alunos que valorizam mais o esforço quanto alunos que valorizam mais a capacidade. Do mesmo modo, no grupo de alunos com mais altos escores na meta evitação do trabalho contam-se também aqueles que valorizam mais o esforço do que a capacidade.

A marcação do peso igual para as duas variáveis por parte da maioria dos alunos poderia ser interpretada como fruto de indecisão ou de falta de conhecimento mais adequado desses conceitos, sem se descartar que a marcação tenha sido, na ocasião, um tanto irrefletida. Também faz sentido o dado que vários alunos voltados à meta evitação tenham declarado que o esforço é decisivo, ao mesmo tempo que eles mesmos não se preocupam com aplicá-lo. Dizem valorizar o esforço, mas, por outras razões, querem e esperam sucesso sem ele.

Entretanto, como explicar que parte dos alunos orientados à meta aprender que, nessa escala, declararam que para o sucesso é preciso esforço, quando questionados sobre o peso de inteligência ou de esforço, qualificaram a inteligência? Esse dado, que parece não dar apoio à teoria de metas de realização, não é absolutamente único, ao aproximar-se do que já apareceu no estudo de Valle e colaboradores (2003), no qual alunos com altos escores na meta aprender também atribuíram o sucesso à capacidade e os insucessos, à falta de esforço.

Numa tentativa de superar a aparente contradição nas respostas verbais de tais alunos, há que se recorrer a um fator cultural, como é sugerido na literatura (Bzuneck, 2001). Isto é, há indicadores em nossa sociedade, com reflexos nos meios acadêmicos, de uma supervalorização generalizada da capacidade em detrimento do esforço. De acordo com essa visão, admitir que um aluno chegou a resultados de aprendizagem pelo esforço aplicado equivale à suposição de que o esforço foi uma estratégia de compensação pela falta de inteligência. Em nossa cultura, mais vale o talento do que a dedicação. Weiner (2004), a propósito de possíveis diferenças culturais em relação a atribuições dos resultados a esforço, aponta que na cultura japonesa o esforço e os seus resultados são mais valorizados do que na cultura norte-americana. Assim, professores japoneses tendem a punir a falta de esforço mais do que professores americanos.

Como conclusão, agora focalizando a presente amostra, diante de uma questão que solicitava um julgamento descontextualizado, pode ter ocorrido a influência cultural, ou seja, ao menos no nível de verbalizações, surgiu uma relativa desvalorização do esforço a favor da capacidade por boa parte dos estudantes da presente amostra, independentemente de sua orientação motivacional. Quando, porém, eles foram solicitados a contextualizar a aplicação do esforço em estratégias numa disciplina específica, apareceu uma coerência plena com a orientação à meta aprender, ao preferirem justamente aquelas que demandam mais esforço, que são as de profundidade. De qualquer forma, vale a sugestão de que novas pesquisas, com instrumentos de medida mais acurados, tragam esclarecimento final sobre o significado das presentes descobertas quanto às crenças sobre esforço.

\section{Percepções do ambiente de aprendizagem}

O ambiente escolar, tal como é percebido pelos alunos, é fator relevante de sua orientação a uma determinada meta de realização (Ames, 1992; Church e colaboradores, 2001; Guimarães, 2004). Nesta pesquisa, do conjunto de variáveis de contexto relativas a uma disciplina específica, foram consideradas as percepções do grau de dificuldade das provas e da carga de trabalho naquela disciplina. Como resultado, considerada a amostra como um todo, surgiram aqui novas e importantes associações.

Assim, ao se relacionarem as metas de realização com a percepção de que as avaliações são exigentes, descobriu-se que houve uma relação discreta entre esse tipo de percepção e a orientação à meta evitação do trabalho. No entanto, foi nula a correlação entre a adoção da meta aprender com esta percepção, um dado semelhante ao que Church e colaboradores (2001) haviam descoberto. Dessa forma, houve uma indicação de que a meta aprender não se desenvolve num contexto psicológico de excessiva pressão nas avaliações. Em relação à percepção de carga de trabalho, encontrou-se na presente amostra uma correlação moderada negativa e significativa com a adoção da meta aprender. Por outro lado, foi positiva e significativa a relação com orientação à meta evitação do trabalho. Entretanto, convém levar em conta os baixos valores de consistência interna entre os itens da escala de percepções. Isso sugere que ela seja aperfeiçoada em novos estudos, a começar pela criação de mais itens para cada objeto de percepção.

No conjunto, os presentes dados relativos às percepções do contexto têm amplo significado para a compreensão dos estados motivacionais dos alunos. Guimarães (2004), apoiada em ampla literatura (por exemplo, Ames, 1992; Greene e colaboradores, 2004), explicita que para a promoção da motivação intrínseca e da meta aprender há uma exigência bem-sustentada quanto ao nível dos desafios que os alunos devem enfrentar, ou seja, entre os extremos de excessivamente difíceis e de muito fáceis. Em outras palavras, a carga de trabalho e as tarefas em geral devem ser difíceis, porém acessíveis, com a expectativa de serem vencidas com o emprego de esforço razoável. Níveis muito altos acarretam ansiedade, enquanto tarefas muito fáceis se tornam tediosas e são deixadas de lado. É com esses parâmetros que se devem apreciar os presentes dados, que apontam que tanto carga de trabalho 
excessiva como avaliações muito pesadas não se associam com adoção da meta aprender. Em particular, percepção de carga excessiva de trabalho está mais relacionada com a meta evitação do trabalho, o que é compatível com as descobertas sobre os efeitos perversos da excessiva dificuldade das tarefas. Em que pese a natureza correlacional dos presentes resultados, é bem plausível a hipótese de que, até certo ponto, há alunos orientados à meta evitação do trabalho por perceberem as tarefas e avaliações como demais exigentes e, assim, menor disposição para um trabalho sério.

\section{Estratégias de aprendizagem e sua relação com as metas de realização}

O último objetivo deste trabalho foi avaliar a adoção de estratégias de superfície e profundidade e relacioná-las com as metas de realização utilizadas pelos alunos da amostra. Os resultados foram consistentes com os de outras pesquisas, descritas por Bzuneck (2005), ou seja, a adoção da meta aprender está relacionada positivamente com o uso de estratégias de profundidade e negativamente com as de superfície, do mesmo modo que a adoção da meta evitação do trabalho se relaciona positivamente com o uso de estratégias de superfície e negativamente com as de profundidade. Adicionalmente, esta pesquisa buscou descobrir o valor de predição das metas adotadas em relação ao tipo de estratégias que são usadas. Pela análise de regressão, novamente os resultados confirmam aquelas associações e estão de acordo com pesquisas anteriores como a de Dupeyrat e Mariné (2001).

Merece comentário a descoberta desta pesquisa de que as estratégias de profundidade, mais associadas à meta aprender, tenham apresentado escores médios similares aos das estratégias de superfície, isto é, os alunos alegaram adotar tanto estratégias de superfície como de profundidade num grau em torno do ponto médio da escala, neste caso com poucas diferenças significativas entre os cursos. A literatura atesta que o uso efetivo de estratégias varia conforme os tipos de tarefas e exigências (Hadwin, Winne, Stockley, Nesbit \& Woszczyna, 2001), ou seja, não se exclui que os mesmos alunos, até com a meta aprender, ora façam uso de um tipo de estratégias ora de outro. Mas os escores médios encontrados sugerem ainda que os alunos da presente amostra tenham conhecimentos limitados acerca de estratégias de aprendizagem. Como argumentaram Wood, Motz e Willoughby (1998), é importante que os alunos conheçam um repertório adequado de estratégias e, mais do que isto, que saibam como e quando utilizá-las. Portanto, o que pode estar ocorrendo é que os estudantes da presente amostra praticam somente algumas estratégias que, de algum modo, aprenderam ao longo do tempo, nas séries anteriores, e que não seriam necessariamente as mais elaboradas, tais como constaram do questionário aqui utilizado.
Em síntese, as presentes descobertas apontam para a necessidade de, em cursos superiores, informar e treinar os alunos quanto a todo o espectro de estratégias de aprendizagem disponíveis. Ao mesmo tempo, os professores devem fazê-los considerar que o esforço desejável nos estudos consiste num envolvimento com estratégias de profundidade.

\section{Referências}

Ames, C. (1992). Classrooms: Goals, structures, and student motivation. Journal of Educational Psychology, 84, 261-271.

Archer, J. (1994). Achievement goals as a measure of motivation in university students. Contemporary Educational Psychology, 19, 430-446.

Biggs, J., Kember, D. \& Leung, D. Y. P. (2001). The revised two-factor Study Process Questionnaire: RSPQ-2F. British Journal of Educational Psychology, 71, 133149.

Bzuneck, J. A. (2001). O esforço nas aprendizagens escolares: mais do que um problema motivacional do aluno. Revista Educação e Ensino, 6, 7-18.

Bzuneck, J. A. (2004a). Aprendizagem por processamento da informação: uma visão construtivista. Em E. Boruchovitch \& J. A. Bzuneck (Orgs.). Aprendizagem: processos psicológicos e o contexto social da escola (pp. 17-54). Petrópolis: Vozes.

Bzuneck, J. A. (2004b). A motivação do aluno orientado a metas de realização. Em E. Boruchovitch, \& J. A. Bzuneck (Orgs.). A motivação do aluno: contribuições da psicologia contemporânea (pp. 58-77). Petrópolis: Vozes.

Bzuneck, J. A. (2005). A motivação dos alunos em cursos superiores. Em M. C. R. A. Joly, A. A. A. dos Santos \& F. F. Sisto (Orgs.). Questôes do cotidiano universitário (pp. 217-237). São Paulo: Casa do Psicólogo.

Bzuneck, J. A., Alves, E., Proietti, H. M. B., Filla, O., L. B., Machado, O. A., Zanatto, R., Machado, R. M. L. \& Araújo, R. V. (2003). Motivação de alunos de cursos superiores: sua relação com envolvimento cognitivo e crenças sobre inteligência. Anais do VI Congresso Brasileiro de Psicologia Escolar e Educacional, Salvador-BA, 238.

Cardoso, L. R. \& Bzuneck, J. A. (2004). Motivação no ensino superior: metas de realização e estratégias de aprendizagem. Psicologia Escolar e Educacional, 8, 145-155.

Church, M. A., Elliot, A. J. \& Gable, S. L. (2001). Perceptions of classroom environment, achievement goals and achievement outcomes. Journal of Educational Psychology, 93, 43-54.

Psico-USF, v. 12, n. 2, p. 291-300, jul./ dez. 2007 
Craik, F. I. M. \& Lockhart, R. S. (1972). Levels of processing: A framework of memory research. Journal of Verbal Learning and Verbal Behavior, 11, 413-418.

Dupeyrat, C. \& Mariné, C. (2001). Implicit theories of intelligence, achievement goals, and learning strategy use. Psychologische Beiträge, 43, 34-52.

Elliott, E. S. \& Dweck, C. S. (1988). Goals: An approach to motivation and achievement. Journal of Personality and Social Psychology, 54, 5-12.

Greene, B. A. \& Miller, R. B. (1996). Influences on achievement goals: Goals, perceived ability and cognitive engagement. Contemporary Educational Psychology, 21, 181192.

Greene, B. A., Miller, R. B., Crowson, H. M., Duke, B. L. \& Akey, K. (2004). Predicting high school cognitive engagement and achievement: Contributions of classroom perceptions and motivation. Contemporary Educational Psychology, 29, 462-482.

Guimarães, S. E. R. (2004). A organização da escola e da sala de aula como determinante da motivação intrínseca e da meta aprender. Em E. Boruchovitch \& J. A. Bzuneck (Orgs.). A motivação do aluno: contribuições da psicologia contemporânea (pp. 78-95). Petrópolis: Vozes.

Hadwin, A. F., Winne, P. H., Stockley, D. B., Nesbit, J. C. \& Woszczyna, C. (2001). Context moderates students' self-reports about how they study. Journal of Educational Psychology, 93, 477-487.

Harackiewicz, J. M., Barron, K. E., Elliot, A. J., Carter, S. M. \& Letho, A. T. (1997). Predictors and consequences of achievement goals in the college classroom: Maintaining interest and making the grade. Journal of Personality and Social Psychology, 73, 1284-1295.

Jacobs, P. A. \& Newstead, S. E. (2000). The nature and development of student motivation. British Journal of Educational Psychology, 70, 243-254.

Marsh, H. W. (2001). Distinguishing between good and bad workloads on students' evaluations of teaching. American Educational Research Journal, 38, 183-212.

Meece, J. (1991). The classroom context and children's motivational goals. Em M. L. Maehr \& P. R. Pintrich (Eds.). Advances in motivation and achievement (pp. 261-289). Greenwich: JAI Press.

Nicholls, J. G. (1984). Achievement motivation: Conceptions of ability, subjective experience, task choice, and performance. Psychological Review, 91, 328-346.

Pintrich, P. R. (2000). An achievement goal theory perspective on issues in motivation terminology, theory, and research. Contemporary Educational Psychology, 25, 92104.

Psico-USF, v. 12, n. 2, p. 291-300, jul./dez. 2007
Pintrich, P. R. \& Garcia, T. (1991). Student goal orientation and self-regulation in college classroom. Em M. L. Maehr \& P. R. Pintrich (Eds.). Advances in motivation and achievement: Goals and self-regulatory processes (pp. 371-402). Greenwich: JAI Press.

Seifert, T. L. \& O'Keefe, B. A. (2001). The relationship of work avoidance and learning goals to perceived competence, externality and meaning. British Journal of Educational Psychology, 71, 81-92.

Stefano, S. R., Passador, J. L. \& Bzuneck, J. A. (2003). As orientações motivacionais em alunos de administração: uma análise realizada na disciplina de Recursos Humanos. Revista Capital Científico, 1, 59-77.

Urdan, T. C. (1997). Achievement goal theory: Past results, future directions. Em M. L. Maehr \& P. R. Pintrich (Eds.). Advances in motivation and achievement (pp. 99-141). Greenwich: JAI Press.

Valle, A., Cabanah, R. G., Nuñez, J. C., González-Pienda, J., Rodríguez, S. \& Piñero, I. (2003). Multiple goals, motivation and academic learning. British Journal of Educational Psychology, 73, 71-87.

Vermetten, Y. J., Lodewijks, H. G. \& Vermunt, J. D. (2001). The role of personality traits and goal orientations in strategy use. Contemporary Educational Psychology, 26, 149-170.

Weiner, B. (2004). Attribution theory revisited: Transforming cultural plurality into theoretical unity. Em D. M. McInerney \& S. V. Ettan (Ed.). Big theories revisited (pp. 13-29). Greenwich: Information Age Publishing.

Weinstein, C. E. \& Mayer, R. E. (1986). The teaching of learning strategies. Em M. C. Wittrock (Ed.). Handbook of research on teaching (pp. 315-327). New York: McMillan.

Wolters, C. A. (2003). Understanding procrastination from self-regulated learning perspective. Journal of Educational Psychology, 95, 179-187.

Wood, E., Motz, M. \& Willoughby, T. (1998). Examining student's retrospective memories of strategy development. Journal of Educational Psychology, 90, 698-704.

Zenorine, R. P. \& Santos, A. A. A. (2003). A motivação e a utilização de estratégias de aprendizagem em universitários. Em E. Mercuri \& S. A. J. Polydoro (Orgs.). Estudante universitário: características e experiências de formação (pp. 67-86). Taubaté-SP: Cabral Universitária.

Recebido em julho de 2006

Reformulado em junho de 2007 Aprovado em agosto de 2007 
Sobre os autores:

Daniella Maculan Pavesi Accorsi é nutricionista com mestrado em Educação pela Universidade Estadual de Londrina - UEL. Foi docente, supervisora de estágio e membro do colegiado do curso de Nutrição da UNOPAR Universidade Norte do Paraná, na qual é atualmente professora dos cursos de pós-graduação em sua área.

José Aloyseo Bzuneck é mestre e doutor pela Universidade de São Paulo em Psicologia Escolar. Foi docente da graduação, especialização e pós-graduação em Educação da Universidade Estadual de Londrina-UEL, onde atua como professor sênior. Pesquisa e publica sobre motivação no contexto escolar, sendo parecerista e membro de conselhos editoriais de revistas científicas na área de Psicologia e Educação.

Sueli Édi Rufini Guimarães é graduada em Psicologia, tem mestrado em Educação pela Universidade Estadual de Londrina e doutorado pela UNICAMP. Atua como docente em cursos de graduação e no Programa de Mestrado em Educação. Coordena projetos de pesquisa, orienta e publica na temática de motivação no contexto escolar. 